East African Medical Journal Vol. 85 No. 5 May 2008

THERAPEUTIC NON-DISCLOSURE OF ADVERSE HEALTH INFORMATION TO AN OBSTETRIC PATIENT: CASE REPORT

O.P. Adudu, FMCA, FWACS, DA, MBBS, Senior Lecturer / Consultant Anaesthesiologist, Department of Anaesthesiology, College of Medical Sciences, University of Benin, P.M.B. 1154, Benin-City, Edo State, Nigeria and O.G. Adudu, FRCS, FWACS, FICS, MBBS, Consultant Surgeon, Department of Surgery, Federal Medical Centre, Asaba, Delta State, Nigeria

Request for reprints to: Dr. O.P. Adudu, Department of Anaesthesiology, College of Medical Sciences, University of Benin, P.M.B. 1154, Benin-City, Edo State, Nigeria

\title{
THERAPEUTIC NON-DISCLOSURE OF ADVERSE HEALTH INFORMATION TO AN OBSTETRIC PATIENT: CASE REPORT
}

\author{
O.P. ADUDU and O.G. ADUDU
}

\begin{abstract}
SUMMARY
The non-disclosure of foetal exomphalos discovered on intra-uterine ultrasound to the mother and anaesthesiologist in this case report, was based on the obstetricians' assumption that it will reduce maternal stress in the antenatal period. There was mis-information that all was well in the antenatal period in spite of maternal informed consent. However, it led to maternal stress and dissatisfaction with the physician. The patient was referred to the psychiatrist for management of her stressful encounter in the postoperative period. Ethical and medico-legal issues raised by therapeutic non disclosure of health information to the patient beforehand was addressed and physician knowledge updated by a review of current literature on the subject.
\end{abstract}

\section{INTRODUCTION}

In every medical specialty including anaesthesia, surgery, obstetrics and gynaecology, bad, sad and difficult information must be given to patients and their families (1). There are also ethical considerations in therapeutic non-disclosure based on the assumption that ignorance is bliss (2). In the patient reported on, potential benefits and harms associated with therapeutic non-disclosure used are discussed. Physician communication style of breaking bad news affects how a physician is perceived, how satisfied recipients of bad news are with the consultation and how they feel after the consultation (3). The case report illustrates these aspects. The literature is replete with disclosure of bad news to oncology patients who have terminal illness (4-9), but only a few addressed the issue of breaking sad and difficult news to patients $(10,11)$ and is non-existent in Africa. This paper is a contribution in this direction and further reviews disclosure and non-disclosure of health information to patients to update current knowledge.

\section{CASE REPORT}

A 32 year old , $82 \mathrm{~kg}$, para $0+0$ female graduate, N.R., was educated preoperatively on the advantages and disadvantages of spinal anaesthesia without sedation for Caesarean section, and the early maternal-child bond developed when baby is put to the breast to suckle after delivery. Patient informed consent for the procedure was obtained. The indication for the Caesarean section was cephalo-pelvic disproportion. Her haemoglobin concentration was $12.6 \mathrm{~g} / \mathrm{dl}$ and the urinalysis was normal. She was assessed as ASA 1. She followed the routine preoperative fasting, instructions.

Intravenous access was established using an 18 guage angiocath and her circulation preloaded with 
1 litre normal saline. The baseline pulse and blood pressure were $72 / \mathrm{min}$ and 120/80 $\mathrm{mmHg}$ (16/10.7 kpa ). She was positioned in the sitting position with her back flexed as far as her gravid uterus allowed. Spinal anaesthesia was established at the L3/L4 interspace and $2.5 \mathrm{ml}$ of $0.5 \%$ heavy plain bupivacaine injected. She was positioned supine and sensory loss tested by pin prick and cold swab, noted to be up to the T8 level after $5 \mathrm{~mm}$. There was associated motor block.

N.R. was cheerful and relaxed during conversation with the anaesthesiologist as the surgery proceeded. On delivery of a live male infant weighing $3.4 \mathrm{~kg}$ and APGAR score of $7 / 1$ and 10/5 min, 10 i.u. of oxytocin i.v. was injected for uterine contraction and decreased haemorrhage. Monitored pulse and blood pressure ranged from $72-84 / \mathrm{min}$ and 100/60-110/ 70 mmHg (14.7/9.3 - 16/10.7 kpa) respectively.

However, the anaesthesiologist discovered to her dismay that the baby after appropriate resuscitation, monitoring and cleaning, could not be transferred to the mother immediately as planned because the obstetricians reported that the mother had no prior knowledge of the foetal exomphalos since intrauterine diagnosis was made and that she was deliberately misinformed that all was well. Consensus was reached to inform her of the baby's abnormality while on the operating table before transfer of the baby was made.

The anaesthesiologist noted that the information was not recorded on the patients chart or was the ultrasound report attached. Surgery and anaesthesia was thereafter uneventful. Blood loss of $800 \mathrm{ml}$ was un-replaced by blood transfusion.

The patient was in stunned silence on disclosure of the information. She was dissatisfied that she had been told the ultrasound report was normal in the antenatal period. She later confided to the anaesthesiologist at the postoperative visit that she was worried her child's abnormality may not resolve as she was told citing the earlier misinformation as the basis for her unbelief.

The patient was therefore educated postoperatively on the need for multiple abdominal surgeries on the infant to encourage closure of the defect underneath the improvised silastic bag. The obstetrician had further discussion on this and the reasons for the therapeutic non-disclosure and deliberate misinformation in the antenatal period when the foetal abnormality was discovered. During discussion, she admitted being traumatised by the news and was referred to the psychiatrist for further management.

Further discussion with the psychiatrist during follow up confirmed mild psychological trauma in the patient which had ameliorated with psychotherapy and needed no anxiolytics.

The abdominal defect had decreased to a great extent underneath the improvised silastic bag following multiple surgical manipulations in the infant at follow up six weeks later.

\section{DISCUSSION}

The main focus of the case report is the therapeutic non disclosure of foetal exomphalos diagnosed ante-natally, to the mother. The obstetricians did not want to increase maternal anxiety (2) and planned that postnatal disclosure would be more beneficial. However, poor physicianphysician communication (12) and documentation resulted in the anaesthesiologist's choice of spinal anaesthesia without sedation for the patient when the obstetricians' had assumed that the patient would have general anaesthesia and would be unaware of her baby's delivery until the postnatal period. This raised ethical issues on doctor-patient communication on prognosis (13) which was good in this case and the risks-benefits of non disclosure when the patient under review had no pre-existing diagnosis of anxiety neurosis.

Also, strategies to effect more appropriate disclosure associated with less harm (2) should have been developed when therapeutic non-disclosure was contemplated in the patient. This should involve a multi-disciplinary team of obstetricians (her primary care physician). anaesthesiologists, paediatricians, and psychiatrists. The primary care physician's professional obligation to his patient is therefore challenging as the perceived insensitive approach which resulted from the poor strategy in this case led to patient distress.

Recognition of these difficulties led to many reported initiatives ranging from increased communication skills training $(5,9,14)$ to the development of guidelines and protocols (14). Baile et al (15) however, commented that breaking bad news was more than just guidelines.

Furthermore, the ethical issue of not exploring the patient's choice between disclosure and non- 
disclosure arose in this case. The need for physician education about patients' rights to choices in giving health information and consent in the environment is therefore important $(12,16,17)$ especially as prognosis was good in this case. The issue of whether non-disclosure was actually therapeutic in this case is a subject for further review.

There is also the need to improve physician communication style which includes patient, disease, and emotion centred communication (18). In this case under review, a patient-centred communication style would have the most positive outcome on a cognitive, evaluative and emotional level which would have decreased her emotional experience (18). Unlike for heart failure patients where the physicians may compromise openness and use euphemisms for the sake of patient's experience based on the well founded fear of upsetting the patient (19), this patient needed open communication as she was a graduate who could easily understand the issues at hand. This view has also been reported by Goldstein et al (13).

The use of deliberate misinformation for the ultrasound report, in the antenatal period has not been reported as a means of non-disclosure during consultation as it is distinct from shielding in cases of patients with serious illness (12) and poor prognosis. It's advantages and disadvantages therefore need further investigation as a communication tool. This investigation should answer the question: "Deliberate misinformation- is it ethically right in an era of informed consent? "Cultural assumptions may have contributed to the obstetricians' decision to deliberately misinform the patient in the antenatal period. This communication style undoubtedly led to a distressful encounter in the patient which was appropriately managed by the psychiatrists.

In conclusion, the therapeutic non disclosure of adverse foetal health information which had a good prognosis, and, deliberate misinformation in this case, raised ethical and medico-legal issues. Strategies that can be developed to reduce stress encountered by the patient when non-disclosure is contemplated were discussed. A review of current literature on disclosure of health information to patients was made. A case is made for education of physicians on communication skills, guidelines and protocols for improved physician-physician communication and disclosure of adverse health information to patients for better management and outcome.

\section{REFERENCES}

1. Fallowfield, L. and Jenkins, V. Communicating sad, bad and difficult news in medicine. Lancet. 2004; 363: 312-319.

2. Berger, J.T. Ignorance is bliss? Ethical considerations in therapeutic non-disclosure (Review). Cancer Investigations. 2005; 23: 94-98.

3. Schmid, M.M., Kindlimann, A. and Langewitz, W. Recipients' perspective on breaking bad news: How you put it really makes a difference. (clinical trial). Pat. Educ. Counsel. 2005; 58: 244-251.

4. Rodriguez, K.L., Gambino, F.J., Butow, P., et al. Pushing up daisies: Implicit and explicit language in oncologist - patient communication about death. Supportive Care in Cancer. 2007; 2: 153-161.

5. Back, A.L., Arnold, R.M., Baile, W.F., et al. Efficacy of communicating skills training for giving bad news and discussing transition to palliative care. Arch. Intern. Med. 2007; 167: 453-460.

6. Eggly, S., Penner, L.A., Greene, M., et al. Information seeking during "bad news" oncology interactions: Question asked by patients and their companions. Soc. Sci. Med. 2006; 63: 2974-2985.

7. Oliffe, I., Thorne, S., Hislop, T.G., et al. "Truth Telling" and cultural assumptions in an era of informed consent. Fam. Comm. Hlth. 2007; 30: 5-15.

8. Moynihan, T.J. and Schapira, L. Preparing ourselves, our trainees and our patients : a commentary on truth telling. J. Clin. Oncol. 2007; 25: 456-457.

9. Alexander, S.C., Keitz, S.A., Sloane, R. and Tulsky, J.A. A controlled trial of a short course to improve communication with patients at the end of life. Aca. Med. 2006; 81: 1008-1012.

10. Ferguson, J.E., $2^{\text {nd }}$, Kleinert, H.L., Lunney, C.A. and Campbell, L.R. Resident physicians competency and attitudes in delivering a postnatal diagnosis of Down Syndrome. Obstet. Gynecol. 2006; 108: 898-905.

11. Davis, R., Davis, B. and Sibert, J. Parents' stories of sensitive and insensitive care by paediatricians in the time leading up to and including diagnostic disclosure of a life limiting condition in their child. Child Care, Hlth. Dev. 2003; 29: 77-82.

12. De, U. and Bhattacharya, P. Communicating bad news: An approach. Indian J. Surg. 2003; 65: 286-289.

13. Goldstein, N.E., Concato, J., Bradley, E.H., et al. Doctor-patient communication about prognosis: The influence of race and financial status. J. Palliative. Med. 2005; 8: 998-1004. 
14. Ungar, L., Alperin, M., Amiel, G.E., et al. Breaking bad news structured training for family medicine residents. Pat. Educ. Counsel. 2003; 48: 63-68.

15. Baile, W.F., Buckman, R., Schapira, L., et al. Breaking bad news: More than just guidelines. J. Oncol. 2006; 24: 3217-3218.

16. WHO: Genomic Resource Centre. World Health Report. Patients' Rights. 2002; 1-3.

17. Evans, K.G. and Henderson, G.L. (eds) In: Consent: A guide for Canadian physicians.CMPA, Ottawa: 2006, $4^{\text {th }}$ edition, $7-11$.
18. Hutsman, R., Visser, A. and Makoul, G. Addressing some of the key questions about communication in health care. Pat. Educ. Counsel. 2005; 58: 221-224.

19. Tayler, M. and Ogden, J. Doctors' use of euphemism and their impact on patients' beliefs about health: An experimental study in patients of heart failure. Pat. Educ. Counsel. 2005; 57: 321-326. 